\title{
A Fully Solid-Phase Synthesis of Biotinylated Glycoclusters
}

\author{
Olivier Renaudet* and Pascal Dumy*
}

Département de Chimie Moléculaire, UMR-CNRS 5250 \& ICMG FR 2607, Université Joseph Fourier, F-38041 Grenoble Cedex 9, France

\begin{abstract}
The fully solid-phase synthesis of chemically well-defined glycoclusters grafted to a topological cyclodecapeptide template is described. The orthogonally protected peptide backbone was first synthesized and cyclized on solid support using D-glutamic acid as first amino acid linked to the resin. After successive regioselective deprotection steps, biotins were coupled to the lower addressable domains of the scaffold, then carbohydrates-binding ligands were assembled as cluster on the upper domain using a chemoselective oxime-based strategy. This provides multitopic labeled glycopeptides which can be easily immobilized to streptavidin-coated surfaces for studying carbohydrate-protein interactions in glycomic researches.
\end{abstract}

\section{INTRODUCTION}

Carbohydrate-protein interactions are closely involved in biological processes including fertilization, cell-cell communication, host-pathogen interactions and cancer metastasis [1-3]. The molecular basis and binding mechanisms which controls these complex recognition events have been largely investigated in the last decade. By contrast with monovalent ligands, it is thus well known that a multivalent display of carbohydrates ensures simultaneous and cooperative contacts with multimeric proteins, increasing thereby the affinity of glycoconjugates owing to the "glycoside cluster effect" [4$6]$. However, the growing number of glycomic researches has highlighted the need of suitable molecular tools enabling the discovery of new effective diagnostic and therapeutic agents [7-9]. Among the prerequisites for exploring such applications is the design of compounds capable of mimicking the multivalent display of the cell surface glycocalix, as well as of being either immobilized or detected in any further biological assays format.

To this end, the typical approach consists in synthesizing well-defined compounds displaying oriented carbohydrate moieties on a molecular scaffold (e.g. calixarenes [10], cyclodextrins [11], dendrimers [12]...). As part of our program related to the evaluation of the interaction between carbohydrates and proteins, we have developed recently a new molecularly well-defined addressable scaffold (Regioselectively Addressable Functionalized Template) [13]. The structural features of this cyclopeptide-based template allow the sequential and regioselective assembly of both biomolecules and any other functional units which provide thereby recognition and effector properties to the molecule [14-17]. Particularly, we have reported the synthesis of diverse multivalent labeled glycoclusters combined solid-phase and solution convergent strategy [18]. The binding properties of biotinylated glycoconjugates with lectins have been recently confirmed using surface plasmon resonance and nanogravimetry experiments [19] after immobilization on streptavidin-coated

*Address correspondence to these authors at the Département de Chimie Moléculaire, UMR-CNRS 5250 \& ICMG FR 2607, Université Joseph Fourier, F-38041 Grenoble Cedex 9, France; E-mail: olivier.renaudet@ujfgrenoble.fr; pascal.dumy@ujf-grenoble.fr surfaces. In order to improve our synthetic method by reducing the number of time-consuming purification steps, we have designed the peptidic template so that a fully supported synthesis, including the cyclization of the peptide, the topological functionalization with biotins and the chemoselective incorporation of sugars which are commonly performed in solution, can be entirely realized on solid-phase (Fig. 1). In this paper, we report this new synthetic procedure providing various biotinylated tetravalent glycoclusters suitable for glycomics.
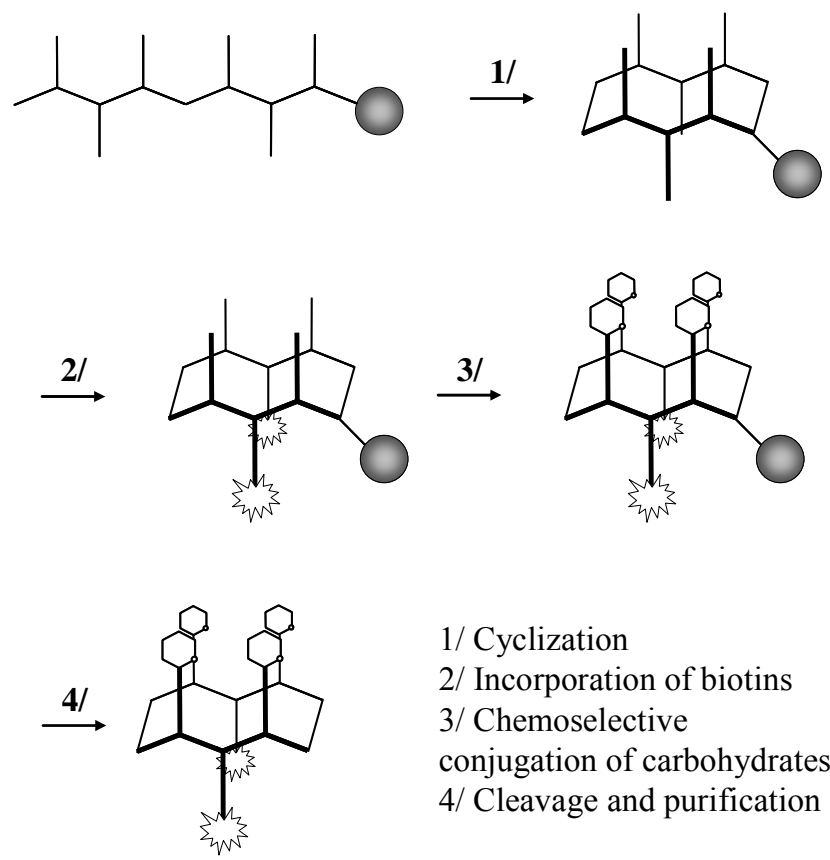

1/ Cyclization
2/ Incorporation of biotins
3/ Chemoselective
conjugation of carbohydrates
4/ Cleavage and purification

Fig. (1). General strategy for the solid-phase synthesis of labeled glycoclusters.

\section{MATERIALS AND METHODOLOGY}

\section{General Information}

All chemical reagents and solvents were purchased from Aldrich (Saint Quentin Fallavier, France) or Acros (Noisy- 
Le-Grand, France) and were used without further purification. Thin layer chromatographies were performed on 0.2 $\mathrm{mm}$ silica 60 coated aluminium foils with F-254 indicator (Merck) and detected under UV light and developed with aqueous sulphuric acid $\left(100 \mathrm{~mL}, \mathrm{H}_{2} \mathrm{SO}_{4} / \mathrm{H}_{2} \mathrm{O} 15 \%\right)$ containing molybdic acid ( $2 \mathrm{~g}$ ) and cerium (IV) sulphate hydrate (1 g). Preparative column chromatographies were done using silica gel (Merck 60, 200-63 $\mu \mathrm{m}) .{ }^{1} \mathrm{H}$ and ${ }^{13} \mathrm{C}$ NMR spectra were recorded on Bruker AC300 spectrometers and chemical shifts $(\delta)$ were reported in parts per million $(\mathrm{ppm})$. Spectra were referenced to the residual proton solvent peaks. Proton and carbon assignments were obtained from GCOSY and ${ }^{1} \mathrm{H}$, ${ }^{13} \mathrm{C}$, GHMQC experiments. Mass spectra were obtained by electron spray ionization on a VG Platform II in the positive mode. Reverse phase HPLC analyses were performed on Waters equipment using $\mathrm{C}_{18}$ columns. The analytical $(\mathrm{Nu}-$ cleosil $120 \AA 3 \mu \mathrm{m} \mathrm{C}_{18}$ particles, $30 \times 4.6 \mathrm{~mm}^{2}$ ) was operated at $1.3 \mathrm{~mL} / \mathrm{min}$ and the preparative (Delta-Pak $300 \AA 15$ $\mu \mathrm{m} \mathrm{C}_{18}$ particles, $200 \times 25 \mathrm{~mm}^{2}$ ) at $22 \mathrm{~mL} / \mathrm{min}$ with $\mathrm{UV}$ monitoring at $214 \mathrm{~nm}$ and $250 \mathrm{~nm}$ using a linear $\mathrm{A}-\mathrm{B}$ gradient (buffer $\mathrm{A}$ : $0.09 \% \mathrm{CF}_{3} \mathrm{CO}_{2} \mathrm{H}$ in water; buffer $\mathrm{B}: 0.09 \%$ $\mathrm{CF}_{3} \mathrm{CO}_{2} \mathrm{H}$ in $90 \%$ acetonitrile).

\section{Synthesis of Cyclodecapeptide (4)}

\section{Synthesis of Linear Peptide (2)}

Linear peptide 2 was synthesized following the Fmoc strategy starting with the Fmoc-Dglu-(OAllyl)-OH 1. Reactions occurred in a glass reaction vessel fitted with a sintered glass frit with the Rink Amide MBHA resin (0.5 g, 0.3 mmol). The resin was swollen with $\mathrm{CH}_{2} \mathrm{Cl}_{2}(10 \mathrm{~mL}, 2 \times 15$ $\mathrm{min})$ and $\mathrm{DMF}(10 \mathrm{~mL}, 1 \times 15 \mathrm{~min})$. The coupling reactions were performed with $N$ - $\alpha$-Fmoc-protected amino acid (0.6 mmol), PyBOP $(0.6 \mathrm{mmol})$ and DIPEA $(1.5 \mathrm{mmol})$ in DMF $(10 \mathrm{~mL})$ for $30 \mathrm{~min}$. After washing with DMF $(10 \mathrm{~mL}, 4 \mathrm{x} 1$ min) and $\mathrm{CH}_{2} \mathrm{Cl}_{2}(10 \mathrm{~mL}, 2 \mathrm{x} 1 \mathrm{~min})$, the completeness of coupling reaction was controlled by Kaiser and TNBS tests. Fmoc protecting groups were removed by treatment with a piperidine/DMF solution (10 $\mathrm{mL}, 1 / 4,3 \times 10 \mathrm{~min})$. After washings with DMF (10 mL, $6 \times 1 \mathrm{~min})$, the completeness of deprotection was controlled by the UV absorption of the piperidine washings at $299 \mathrm{~nm}$ (loading measured: 0.47 $\mathrm{mmol} / \mathrm{g})$.

\section{Synthesis of Linear Peptide (3)}

The resin bearing the linear peptide $2(0.2 \mathrm{~g}, 0.12 \mathrm{mmol})$ was swollen with $\mathrm{CH}_{2} \mathrm{Cl}_{2}$ (10 mL, $\left.2 \times 15 \mathrm{~min}\right)$ and DMF (10 $\mathrm{mL}, 1 \times 15 \mathrm{~min})$ then treated with $\mathrm{PhSiH}_{3}(370 \mu \mathrm{L}, 3 \mathrm{mmol})$ in dry $\mathrm{CH}_{2} \mathrm{Cl}_{2}(5 \mathrm{~mL})$ for 5 minutes. $\mathrm{Pd}\left(\mathrm{Ph}_{3} \mathrm{P}\right)_{4}(56 \mathrm{mg}, 0.05$ mmol) was added and the resin was stirred under argon gas for 20 minutes. The reagents were removed by filtration, the resin washed with $\mathrm{CH}_{2} \mathrm{Cl}_{2}(10 \mathrm{~mL}, 4 \times 1 \mathrm{~min})$ and the procedure was repeated. The resin was finally washed with $\mathrm{CH}_{2} \mathrm{Cl}_{2}$ (10 mL, 2x1 min), a solution of dioxane/water (10 $\mathrm{mL}, 9 / 1,2 \times 1 \mathrm{~min})$ and $\mathrm{DMF}(10 \mathrm{~mL}, 2 \times 1 \mathrm{~min})$ then treated three times with a solution of piperidine in DMF $(5 \mathrm{~mL}, 1 / 4$, $10 \mathrm{~min})$ and washed with DMF (10 mL, 6x1 min). The completeness of deprotection was controlled by the UV absorption of the piperidine washings at $299 \mathrm{~nm}$ (loading measured: $0.4 \mathrm{mmol} / \mathrm{g}$ ).

\section{Cyclization (4)}

The resin $3(0.12 \mathrm{mmol})$ was swollen with $\mathrm{CH}_{2} \mathrm{Cl}_{2}(2 \times 15$ minutes) and DMF (1x15 min). PyAOP (125 mg, 0.24 mmol) and DIPEA (104 $\mu \mathrm{L}, 0.6 \mathrm{mmol})$ were added the resin in DMF ( $5 \mathrm{~mL}$ ) and the mixture was stirred at room temperature. After 30 minutes, the reagents were removed by filtration, the resin washed with DMF (2x1 min) then the procedure was repeated. The completeness of coupling reaction was confirmed by both Kaiser and TNBS tests. An aliquot of resin was taken out and treated with pure TFA for 20 minutes then the filtrate was analyzed. RP-HPLC: $\mathrm{R}_{\mathrm{t}}=31.0 \mathrm{~min}$ (linear gradient: $95: 5$ to $0: 100 \mathrm{~A}: \mathrm{B}$ in 30 minutes; detection: $\lambda=214$ and $250 \mathrm{~nm}$ ). MS-ESI (positive mode): calcd for $\mathrm{C}_{105} \mathrm{H}_{141} \mathrm{~N}_{21} \mathrm{O}_{31} 2193.4, \mathrm{~m} / \mathrm{z}:[\mathrm{M}+3 \mathrm{H}]^{3+}=731.5,[\mathrm{M}+2 \mathrm{H}]^{2+}=$ 1096.9.

\section{Synthesis of Biotinylated Cyclodecapeptide (6)}

\section{Synthesis of Biotinylated Peptide (5)}

The resin 4 (0.12 mmol estimated) was swollen with $\mathrm{CH}_{2} \mathrm{Cl}_{2}$ (2x15 minutes) and DMF (1x15 min). To remove Dde, it was treated with a solution of hydrazine in DMF (10 $\mathrm{mL}, 1 / 24,4 \times 10 \mathrm{~min}$ ) then washed successively with DMF (10 $\mathrm{mL}, 4 \times 1 \mathrm{~min}), \mathrm{CH}_{2} \mathrm{Cl}_{2}(10 \mathrm{~mL}, 2 \times 1 \mathrm{~min})$ and $\mathrm{DMF}(10$ $\mathrm{mL}, 2 \mathrm{x} 1 \mathrm{~min})$. The resulting resin $(0.12 \mathrm{mmol})$ was then stirred in the presence biotin (117 $\mathrm{mg}, 0.48 \mathrm{mmol})$, PyBOP (250 $\mathrm{mg}, 0.48 \mathrm{mmol})$ and DIPEA $(308 \mu \mathrm{L}, 1.2 \mathrm{mmol})$ in DMF $(5 \mathrm{~mL})$. After 1 hour, the solution was filtrated and the resin washed with DMF $(10 \mathrm{~mL}, 4 \times 1 \mathrm{~min})$ and $\mathrm{CH}_{2} \mathrm{Cl}_{2}(10$ $\mathrm{mL}, 2 \mathrm{x} 1 \mathrm{~min})$. This procedure was repeated once. The completeness of coupling reaction was confirmed by both Kaiser and TNBS tests to obtain 5. An aliquot of resin was taken out and treated with pure TFA for 20 minutes for RP-HPLC analysis. RP-HPLC: $\mathrm{R}_{\mathrm{t}}=26.5$ min (linear gradient: $95: 5$ to 0:100 A:B in 30 minutes; detection: $\lambda=214$ and $250 \mathrm{~nm}$ ). Estimated yield for 5 (by RP-HPLC): $80 \%$.

\section{Incorporation of Methyl Ketones (6)}

The resin 5 (0.12 mmol estimated) was swollen with $\mathrm{CH}_{2} \mathrm{Cl}_{2}$ (2x15 minutes) and DMF (1x15 min). PnZ protecting groups were removed from 5 by using a solution containing $\mathrm{SnCl}_{2}(2 \mathrm{M})$, acetic acid $(1.6 \mathrm{mM})$, phenol $(10 \mathrm{mM})$ in $\mathrm{DMF}(5 \mathrm{~mL})$ for 1 hour at room temperature. The procedure was repeated three times and the resin was washed successively with DMF, $\mathrm{CH}_{2} \mathrm{Cl}_{2} /$ triethylamine (9/1), DMF and $\mathrm{CH}_{2} \mathrm{Cl}_{2}$. Pentofluorophenol levulinic ester $(270 \mathrm{mg}, 0.96$ $\mathrm{mmol})$ was coupled to the peptide in DMF $(5 \mathrm{~mL})$ in the presence of DIPEA $(83 \mu \mathrm{L}, 0.48 \mathrm{mmol})$. After $30 \mathrm{~min}$, the solution was filtrated and the resin washed successively with DMF (10 mL, 4x1 min) and $\mathrm{CH}_{2} \mathrm{Cl}_{2}(10 \mathrm{~mL}, 2 \times 1 \mathrm{~min})$. The procedure was repeated until completeness of the reaction (controlled by Kaiser and TNBS tests). An aliquot of resin was taken out and treated with pure TFA for 20 minutes for RP-HPLC analysis. RP-HPLC: $\mathrm{R}_{\mathrm{t}}=17.3$ min (linear gradient: $95: 5$ to $0: 100 \mathrm{~A}: \mathrm{B}$ in 30 minutes; detection: $\lambda=214$ and $250 \mathrm{~nm}$ ). Estimated yield for 6 (by RP-HPLC): $50 \%$.

\section{O-(2,3,4,6-tetra-O-benzyl- $\alpha$-D-galactopyranosyl)-N- oxyphthalimide $(8)$}

To a stirring solution of galactosyl fluoride donor 7 (1.02 g, $1.9 \mathrm{mmol})$ in $\mathrm{CH}_{2} \mathrm{Cl}_{2}(20 \mathrm{~mL}), N$-hydroxyphthalimide $(0.306 \mathrm{~g}, 1.9 \mathrm{mmol})$, triethylamine $(261 \mu \mathrm{L}, 1.9 \mathrm{mmol})$ and 
$\mathrm{BF}_{3} \cdot \mathrm{Et}_{2} \mathrm{O}(1.14 \mathrm{~mL}, 9 \mathrm{mmol})$ were added. After 1 hour, the organic layer was washed twice with $10 \%$ aqueous sodium hydrogenocarbonate then water. The organic layer was dried over $\mathrm{Na}_{2} \mathrm{SO}_{4}$ and evaporated. After flash chromatography on silica gel $\left(\mathrm{CH}_{2} \mathrm{Cl}_{2}\right.$ /ethyl acetate, 9/1), both alpha and beta anomer were separated. Compound 8 (alpha anomer) was obtained $(0.8 \mathrm{~g}, 62 \%)$ was obtained as a colorless oil. ${ }^{1} \mathrm{H}$ NMR (300 MHz, $\mathrm{CDCl}_{3}$ ): $\delta=7.88-7.71$ (m, 4H, Har.pht), 7.64-7.29 (m, 20H, Har. ${ }_{\text {Bn }}$ ), $5.75\left(\mathrm{~d}, 1 \mathrm{H},{ }^{3} \mathrm{~J}_{1,2}=3.8 \mathrm{~Hz}, \mathrm{H}-1\right)$, 5.18-4.54 (m, 8H, 4CH $)$, 5.02-4.99 (m, 1H, H-5), 4.37 (dd, $\left.1 \mathrm{H},{ }^{3} \mathrm{~J}_{1,2}=3.8 \mathrm{~Hz},{ }^{3} \mathrm{~J}_{2,3}=10.6 \mathrm{~Hz} \mathrm{H}-2\right), 4.25-4.20(\mathrm{~m}, 2 \mathrm{H}, \mathrm{H}-$ 3, H-4), 3.74-3.60 (m, 2H, H-6). ${ }^{13} \mathrm{C}$ NMR (75 MHz, $\left.\mathrm{CDCl}_{3}\right): \delta=163.8(\mathrm{C}=\mathrm{O}), 139.2\left(\mathrm{Car}_{\cdot \mathrm{Bn}}\right), 139.0$ (Car. $\left.{ }_{\mathrm{Bn}}\right)$, 138.7 (Car.Bn), 138.5 (Car.Bn), 134.8 (CHar.Pht), 129.4 (Car.Pht), 129.0 (CHar. ${ }_{\text {Bn }}$ ), 128.9 (CHar. ${ }_{\text {Bn }}$ ), 128.8 (CHar. ${ }_{\text {Bn }}$ ), 128.7 (CHar. ${ }_{\text {Bn }}$ ), 128.7 (CHar. ${ }_{\text {Bn }}$ ), 128.6 (CHar. ${ }_{\text {Bn }}$ ), 128.6 (CHar. $\left.{ }_{\text {Bn }}\right), 128.5$ (CHar.Bn), 128.3 (CHar. $\left.{ }_{\text {Bn }}\right), 128.2$ (CHar. ${ }_{\text {Bn }}$ ), 128.1 (CHar. $_{\cdot \mathrm{Bn}}$ ), 128.0 (CHar. ${ }_{\mathrm{Bn}}$ ), 127.9 (CHar.Bn), 127.9 (CHar.Bn), 123.9 (CHar.Pht), 103.1 (C-1), 78.6, 75.9 (C-3, C4), $75.5(\mathrm{C}-2), 75.4\left(\mathrm{CH}_{2}\right), 73.9\left(\mathrm{CH}_{2}\right), 73.7\left(\mathrm{CH}_{2}\right), 73.3$ $\left(\mathrm{CH}_{2}\right), 71.4$ (C-5), 68.6 (C-6). MS-ESI (positive mode): calcd for $\mathrm{C}_{42} \mathrm{H}_{40} \mathrm{NO}_{8} 686.27[\mathrm{M}+\mathrm{H}]^{+}$, found: 686.10. Beta anomer (174 mg, 25\%): ${ }^{1} \mathrm{H}$ NMR $\left(300 \mathrm{MHz}, \mathrm{CDCl}_{3}\right): \delta=$ 7.91-7.72 (m, 4H, Har.Pht), 7.60-7.23 (m, 20H, Har.Bn), 5.26$4.65\left(\mathrm{~m}, 8 \mathrm{H}, 4 \mathrm{CH}_{2}\right), 5.11\left(\mathrm{~d}, 1 \mathrm{H},{ }^{3} \mathrm{~J}_{1,2}=7.9 \mathrm{~Hz}, \mathrm{H}-1\right), 4.19(\mathrm{t}$, $\left.1 \mathrm{H},{ }^{3} \mathrm{~J}_{2,3}=7.9 \mathrm{~Hz}, \mathrm{H}-2\right), 3.92$, (bd, $1 \mathrm{H},{ }^{3} \mathrm{~J}_{3,4}={ }^{3} \mathrm{~J}_{4,5}=2.3 \mathrm{~Hz}$, $\mathrm{H}-4), 3.70-3.63$ (m, 4H, H-3, H-5, H-6). ${ }^{13} \mathrm{C}$ NMR $(75 \mathrm{MHz}$, $\left.\mathrm{CDCl}_{3}\right): \delta=163.3(\mathrm{C}=\mathrm{O}), 138.9\left(\mathrm{Car}_{\mathrm{Bn}}\right), 138.8$ (Car. $\left.{ }_{\mathrm{Bn}}\right)$, 138.4 (Car.Bn), 134.8 (CHar.Pht), 129.4 (Car.Pht), 128.9

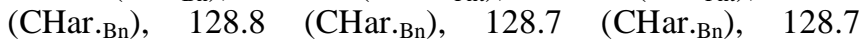
(CHar.Bn), $128.6 \quad$ (CHar.Bn), $128.6 \quad$ (CHar. $\left.{ }_{\text {Bn }}\right), \quad 128.1$ (CHar. $\left.{ }_{\text {Bn }}\right), \quad 128.0 \quad$ (CHar. $\left.{ }_{\text {Bn }}\right), \quad 128.0 \quad$ (CHar. $\left.{ }_{\text {Bn }}\right), \quad 124.0$ (CHar.Pht), 108.8 (C-1), 82.3 (C-3 ou C-5), 78.3 (C-2), 75.8 $\left(\mathrm{CH}_{2}\right), 75.0\left(\mathrm{CH}_{2}\right), 74.9(\mathrm{C}-3$ ou C-5 $), 73.9\left(\mathrm{CH}_{2}\right), 73.9(\mathrm{C}-$ 4), $73.8\left(\mathrm{CH}_{2}\right), 69.2$ (C-6). MS-ESI (positive mode): calcd for $\mathrm{C}_{42} \mathrm{H}_{40} \mathrm{NO}_{8} 686.27[\mathrm{M}+\mathrm{H}]^{+}$, found: 686.87 .

\section{O- $\alpha$-D-galactopyranosyl oxyamine (9)}

Compound 8 (0.78 g, $1.1 \mathrm{mmol})$ was debenzylated by hydrogenation over $\mathrm{Pd} / \mathrm{C} \quad 10 \% \quad(0.1 \mathrm{~g}, 0.9 \mathrm{mmol})$ in $\mathrm{MeOH} / \mathrm{CH}_{2} \mathrm{Cl}_{2}(10 \mathrm{~mL}, 1 / 1)$ under hydrogen atmosphere. After 4 hours, the $\mathrm{Pd} / \mathrm{C}$ was removed by filtration through celite and the filtrate was evaporated to dryness. The resulting oil containing partially debenzylated intermediates was purified by flash chromatography. The fully debenzylated compound was finally treated overnight with a solution of methylhdyrazine/ethanol $(1 / 1,10 \mathrm{~mL})$. After evaporation to dryness, the aminooxy alpha galactosyl 9 was obtained as a white solid by precipitation with $\mathrm{MeOH} / \mathrm{CH}_{2} \mathrm{Cl}_{2}(0.12 \mathrm{~g}$, $55 \%) .{ }^{1} \mathrm{H}$ NMR $\left(300 \mathrm{MHz}, \mathrm{D}_{2} \mathrm{O}\right): \delta=5.11\left(\mathrm{~d}, 1 \mathrm{H},{ }^{3} \mathrm{~J}_{1,2}=3.9\right.$ $\mathrm{Hz}, \mathrm{H}-1)$, 4.07-4.00 (m, 2H, H-6), 3.93 (dd, $1 \mathrm{H},{ }^{3} \mathrm{~J}_{1,2}=3.9$ $\left.\mathrm{Hz},{ }^{3} \mathrm{~J}_{2,3}=10.4 \mathrm{~Hz}, \mathrm{H}-2\right)$, 3.89-3.78 (m, 3H, H-3, H-4, H-5). ${ }^{13} \mathrm{C}$ NMR $\left(75 \mathrm{MHz}, \mathrm{D}_{2} \mathrm{O}\right): \delta=102.0(\mathrm{C}-1), 71.4,69.7,69.6$, 68.2 (C-2, C-3, C-4, C-5), 61.5 (C-6). MS-ESI (positive mode): calcd for $\mathrm{C}_{6} \mathrm{H}_{14} \mathrm{NO}_{6} 196.08[\mathrm{M}+\mathrm{H}]^{+}$, found: 196.10 .

\section{O- $\beta$-D-galactopyranosyl oxyamine (10)}

A similar procedure was followed for the preparation of 10. ${ }^{1} \mathrm{H}$ NMR (300 MHz, $\left.\mathrm{D}_{2} \mathrm{O}\right): \delta=4.54\left(\mathrm{~d}, 1 \mathrm{H},{ }^{3} \mathrm{~J}_{1,2}=8.1\right.$ $\mathrm{Hz}, \mathrm{H}-1$ ), 3.94 (bd, $\left.1 \mathrm{H},{ }^{3} \mathrm{~J}_{3,4}=3.4 \mathrm{~Hz}, \mathrm{H}-4\right), 3.87-3.73$ (m, $3 \mathrm{H}, \mathrm{H}-5, \mathrm{H}-6), 3.69$ (dd, $\left.1 \mathrm{H},{ }^{3} \mathrm{~J}_{2,3}=9.8 \mathrm{~Hz}, \mathrm{H}-3\right), 3.55$ (dd, $\left.1 \mathrm{H},{ }^{3} \mathrm{~J}_{1,2}=8.1 \mathrm{~Hz},{ }^{3} \mathrm{~J}_{2,3}=9.8 \mathrm{~Hz}, \mathrm{H}-2\right) .{ }^{13} \mathrm{C} \mathrm{NMR}(75 \mathrm{MHz}$,
$\left.\mathrm{D}_{2} \mathrm{O}\right): \delta=105.9(\mathrm{C}-1), 75.5(\mathrm{C}-3), 73.2$ (C-2), 69.7 (C-4), 69.0 (C-5), 61.4 (C-6). MS-ESI (positive mode): calcd for $\mathrm{C}_{6} \mathrm{H}_{14} \mathrm{NO}_{6} 196.08[\mathrm{M}+\mathrm{H}]^{+}$, found: 196.00.

\section{O- $\alpha$-D-mannopyranosyl oxyamine (11)}

A similar procedure was followed for the preparation of 11. ${ }^{1} \mathrm{H}$ NMR $\left(300 \mathrm{MHz}, \mathrm{D}_{2} \mathrm{O}\right): \delta=5.00\left(\mathrm{~d}, 1 \mathrm{H}, \mathrm{J}_{1,2}=1.7 \mathrm{~Hz}\right.$, $\mathrm{H}-1), 4.03$ (dd, $\left.1 \mathrm{H}, \mathrm{J}_{1,2}=1.7 \mathrm{~Hz}, \mathrm{~J}_{2,3}=2.8 \mathrm{~Hz}, \mathrm{H}-2\right), 3.97$ $\left(\mathrm{dd}, 1 \mathrm{H}, \mathrm{J}_{5,6 \mathrm{a}}=1.3 \mathrm{~Hz}, \mathrm{~J}_{6 \mathrm{a}, 6 \mathrm{~b}}=12.3 \mathrm{~Hz}, \mathrm{H}-6 \mathrm{a}\right), 3.88\left(\mathrm{dd}, \mathrm{J}_{5,6 \mathrm{~b}}\right.$ $\left.=3.7 \mathrm{~Hz}, \mathrm{~J}_{6 \mathrm{a}, 6 \mathrm{~b}}=12.3 \mathrm{~Hz}, \mathrm{H}-6 \mathrm{~b}\right), 3.76-3.72(\mathrm{~m}, 3 \mathrm{H}, \mathrm{H}-3, \mathrm{H}-$ 4, H-5); ${ }^{13} \mathrm{C}$ NMR (75 MHz, D $\left.2 \mathrm{O}\right): \delta=103.8$ (C-1), 73.4, 71.2, 69.4 (C-2), 67.2, 61.4 (C-6). MS-CI (positive mode): calcd for $\mathrm{C}_{6} \mathrm{H}_{14} \mathrm{NO}_{6} 196.08[\mathrm{M}+\mathrm{H}]^{+}$, found: 195.94 .

\section{O-(2-acetamido)- $\alpha$-D-galactopyranosyl oxyamine (12)}

A similar procedure was followed for the preparation of 12. ${ }^{1} \mathrm{H}$ NMR (300 MHz, $\left.\mathrm{D}_{2} \mathrm{O}\right): \delta=4.99\left(\mathrm{~d}, 1 \mathrm{H}, \mathrm{J}_{1,2}=4.1 \mathrm{~Hz}\right.$, $\mathrm{H}-1), 4.24$ (dd, $\left.1 \mathrm{H}, \mathrm{J}_{1,2}=4.1 \mathrm{~Hz}, \mathrm{~J}_{2,3}=11.3 \mathrm{~Hz}, \mathrm{H}-2\right), 4.03-$ 3.99 (m, 2 H, H-4, H-5), 3.89-3.76 (m, 3 H, H-3, H-6), 2.08 $\left(\mathrm{s}, 3 \mathrm{H}, \mathrm{HNCOCH}_{3}\right) .{ }^{13} \mathrm{C}$ NMR $\left(75 \mathrm{MHz}, \mathrm{D}_{2} \mathrm{O}\right): \delta=175.0$ $\left(\mathrm{HNCOCH}_{3}\right), 101.0(\mathrm{C}-1), 71.4,68.8$ (C-4, C-5), 68.0 (C-3), $61.5(\mathrm{C}-6), 49.6(\mathrm{C}-2), 22.3\left(\mathrm{HNCOCH}_{3}\right)$. MS-CI (positive mode): calcd for $\mathrm{C}_{8} \mathrm{H}_{17} \mathrm{~N}_{2} \mathrm{O}_{6} 237.10[\mathrm{M}+\mathrm{H}]^{+}$, found: 237.00 .

\section{O-( $\beta$-D-galactopyranosyl $)-\left(1 \rightarrow 4^{\prime}\right)-(\beta$-D-glucopyranosyl $)$ oxyamine (13)}

A similar procedure was followed for the preparation of 13. ${ }^{1} \mathrm{H}$ NMR $\left(300 \mathrm{MHz}, \mathrm{D}_{2} \mathrm{O}\right): \delta=4.62\left(\mathrm{~d}, 1 \mathrm{H}, \mathrm{J}_{1^{\prime}, 2},=8.3\right.$ Hz, H-1'), 4.47 (d, $1 \mathrm{H}, \mathrm{J}_{1,2}=7.7 \mathrm{~Hz}, \mathrm{H}-1$ ), 4.03 (dd, $1 \mathrm{H}$, $\left.\mathrm{J}_{5^{\prime}, 6 \mathrm{a}^{\prime}}=1.3 \mathrm{~Hz}, \mathrm{~J}_{6 \mathrm{a}^{\prime}, 6 \mathrm{~b}^{\prime}}=11.6 \mathrm{~Hz}, \mathrm{H}-6 \mathrm{a}^{\prime}\right), 3.95\left(\mathrm{bd}, 1 \mathrm{H}, \mathrm{J}_{3,4}=\right.$ $3.1 \mathrm{~Hz}, \mathrm{H}-4), 3.84$ (dd, $1 \mathrm{H}, \mathrm{J}_{5^{\prime}, 6 \mathrm{~b}^{\prime}}=4.6 \mathrm{~Hz}, \mathrm{~J}_{6 \mathrm{a}^{\prime}, 6 \mathrm{~b}^{\prime}}=11.6 \mathrm{~Hz}$, H-6b'), 3.79-3.63 (m, 7 H, H-3, H-5, H-6, H-3', H-4', H-5'), $3.66\left(\mathrm{dd}, 1 \mathrm{H}, \mathrm{J}_{1,2}=7.7 \mathrm{~Hz}, \mathrm{~J}_{2,3}=9.8 \mathrm{~Hz}, \mathrm{H}-2\right), 3.38$ (bt, $1 \mathrm{H}$, $\left.\mathrm{J}_{2}, 3^{\prime}=8.4 \mathrm{~Hz}, \mathrm{H}-2^{\prime}\right) .{ }^{13} \mathrm{C} \mathrm{NMR}\left(75 \mathrm{MHz}, \mathrm{D}_{2} \mathrm{O}\right): \delta=105.2$ (C-1'), 103.3 (C-1), 78.6, 75.7, 75.1, 74.8, 72.8, 71.7 (C-2'), 71.3 (C-2), 68.9 (C-4), 61.4 (C-6’), 60.4 (C-6). ES-MS (positive mode): calcd for $\mathrm{C}_{12} \mathrm{H}_{24} \mathrm{NO}_{11} 358.13[\mathrm{M}+\mathrm{H}]^{+}$, found: 358.15 .

\section{Synthesis of Biotinylated Glycoclusters (14-18)}

The resin 6 ( $4 \mu \mathrm{mol}$ estimated) was swollen with $\mathrm{CH}_{2} \mathrm{Cl}_{2}$ (2x15 minutes) and DMF (1x15 min). Aminooxy sugars 9$13(64 \mu \mathrm{mol})$ were added to the resin bearing peptide 6 in $\mathrm{DMF} / \mathrm{AcOH} / \mathrm{H}_{2} \mathrm{O}(1 / 1 / 0.1 ; 1 \mathrm{~mL})$. The resin was stirred at room temperature overnight. After successive washings with $\mathrm{DMF} / \mathrm{AcOH} / \mathrm{H}_{2} \mathrm{O}(1 / 1 / 0.1 ; 2 \times 1 \mathrm{~min}), \mathrm{DMF}(2 \times 1 \mathrm{~min})$ and $\mathrm{CH}_{2} \mathrm{Cl}_{2}$ (2x1 min), the glycoclusters were finally cleaved from the resin with pure TFA $(1 \mathrm{~h})$. After purification by semi-preparative RP-HPLC (linear gradient: 95:5 to 40:60 $\mathrm{A}: \mathrm{B}$ in 30 minutes; detection: $\lambda=214$ and $250 \mathrm{~nm}$ ) the glycoclusters 14-18 were obtained as white powders (2 mg) with 5-10\% overall yield. 14: RP-HPLC: $\mathrm{R}_{\mathrm{t}}=18.3$ min (linear gradient: $95: 5$ to $40: 60 \mathrm{~A}: \mathrm{B}$ in 30 minutes; detection: $\lambda=214$ and $250 \mathrm{~nm}$ ). MS-ESI (positive mode): calcd for $\mathrm{C}_{117} \mathrm{H}_{193} \mathrm{~N}_{25} \mathrm{O}_{43} \mathrm{~S}_{2}$ 2700.31, m/z: $[\mathrm{M}+3 \mathrm{H}]^{3+}=901.4$, $[\mathrm{M}+2 \mathrm{H}]^{2+}=1352.0$. 15: RP-HPLC: $\mathrm{R}_{\mathrm{t}}=18.7 \mathrm{~min}$ (linear gradient: $95: 5$ to $40: 60 \mathrm{~A}: \mathrm{B}$ in 30 minutes; detection: $\lambda=214$ and $250 \mathrm{~nm}$ ). MS-ESI (positive mode): calcd for $\mathrm{C}_{117} \mathrm{H}_{193} \mathrm{~N}_{25} \mathrm{O}_{43} \mathrm{~S}_{2}$ 2700.31, m/z: $[\mathrm{M}+3 \mathrm{H}]^{3+}=901.2$, $[\mathrm{M}+2 \mathrm{H}]^{2+}=1352.4$. 16: RP-HPLC: $\mathrm{R}_{\mathrm{t}}=18.5 \mathrm{~min}$ (linear gradient: $95: 5$ to $40: 60 \mathrm{~A}: \mathrm{B}$ in 30 minutes; detection: $\lambda=214$ and $250 \mathrm{~nm}$ ). MS-ESI (positive mode): calcd for 
$\mathrm{C}_{117} \mathrm{H}_{193} \mathrm{~N}_{25} \mathrm{O}_{43} \mathrm{~S}_{2}$ 2700.31, m/z: $[\mathrm{M}+3 \mathrm{H}]^{3+}=$ 901.4, $[\mathrm{M}+2 \mathrm{H}]^{2+}=1351.9$. 17: RP-HPLC: $\mathrm{R}_{\mathrm{t}}=18.9$ min (linear gradient: $95: 5$ to $40: 60 \mathrm{~A}: \mathrm{B}$ in 30 minutes; detection: $\lambda=214$ and $250 \mathrm{~nm}$ ). MS-ESI (positive mode): calcd for $\mathrm{C}_{125} \mathrm{H}_{205} \mathrm{~N}_{29} \mathrm{O}_{43} \mathrm{~S}_{2}$ 2864.41, m/z: $[\mathrm{M}+3 \mathrm{H}]^{3+}=956.2$, $[\mathrm{M}+2 \mathrm{H}]^{2+}=1434.3$. 18: RP-HPLC: $\mathrm{R}_{\mathrm{t}}=18.9$ min (linear gradient: $95: 5$ to $40: 60 \mathrm{~A}: \mathrm{B}$ in 30 minutes; detection: $\lambda=214$ and $250 \mathrm{~nm}$ ). MS-ESI (positive mode): calcd for $\mathrm{C}_{141} \mathrm{H}_{233} \mathrm{~N}_{25} \mathrm{O}_{63} \mathrm{~S}_{2} \quad 3348.52, \quad \mathrm{~m} / \mathrm{z}: \quad[\mathrm{M}+3 \mathrm{H}]^{3+}=1117.8$, $[\mathrm{M}+2 \mathrm{H}]^{2+}=1679.2$.

\section{RESULTS AND DISCUSSION}

The development of polymer-supported synthetic methodologies has considerably promoted the access to bioconjugates. It requires a careful choice of solid support as well as suitable orthogonal protecting groups for amino acids to ensure the controlled assembly and the regioselective functionalization of the peptide. Our cyclic scaffold has been designed on the basis of the Template Assembled Synthetic Protein (TASP) model which was previously described for protein de novo design [20]. It exhibits two independent addressable domains displaying lysine residues as anchoring sites. We have selected para-nitrobenzyl-oxycarbonyl (pNZ) [21] and 1-(4,4-dimethyl-2,6-dioxocyclohex-1-ylidene)ethyl (Dde) [22] as orthogonal protecting groups for lysine sidechain to enable the regioselective conjugation with carbohydrates and biotins.

Besides this, it was shown that oxime ligation represents a highly efficient method for conjugation of various biomolecules [23-26]. Particularly, we and others have reported that aminooxy function can be easily incorporated to anomer position of sugars [27-29] and that this modification enables the quantitative coupling to peptide containing either aldehyde or ketone moieties [18, 30, 31]. In addition, in respect with previous studies showing the stability of oxime linkage in acidic conditions, we chose the acido-labile Rink-Amide MBHA $^{\circledast}$ resin which is expected to be compatible with all further the experimental conditions.

The decapeptide 2 (Fig. 2), linear precursor of the cyclic template 4, was assembled following the standard Fmoc protocol by using activation with PyBOP/DIPEA (benzotriazole-1-yloxytripyrrolidino phosphoniumhexafluorophosphate/diisopropyletylamine) [32]. Noteworthy, we used Dglutamic acid derivative $\mathbf{1}$ as attachment site on the resin to secure the convenient conformation of the template [33]. The removal of Allyl then 9-fluorenylmethoxycarbonyl (Fmoc) protecting group was achieved by typical procedure using

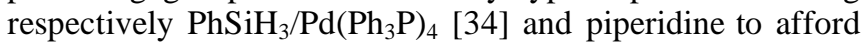
the linear peptide 3 presenting free $N$ - and $C$-terminal end.

The head-to-tail cyclization of the template is a critical step that should occur cleanly and quantitatively to prevent the possible formation of truncated structures during further functionalizations. Thus, this reaction was carefully controlled by Kaiser [35] and 2,4,6-trinitrobenzene sulfonic acid (TNBS) [36] tests with a few beads of resin. After two reaction cycles using PyAOP as coupling reagent [37] and DIPEA as base, both colorimetric tests were negative, denoting the completeness of the cyclization. To confirm this observation, an aliquot of resin was treated with trifluoroacetic acid (TFA) and the resulting solution analyzed by reversephase HPLC and mass spectrometry. These analyses have shown a clean crude reaction mixture since neither linear peptide nor truncated structures have been detected.
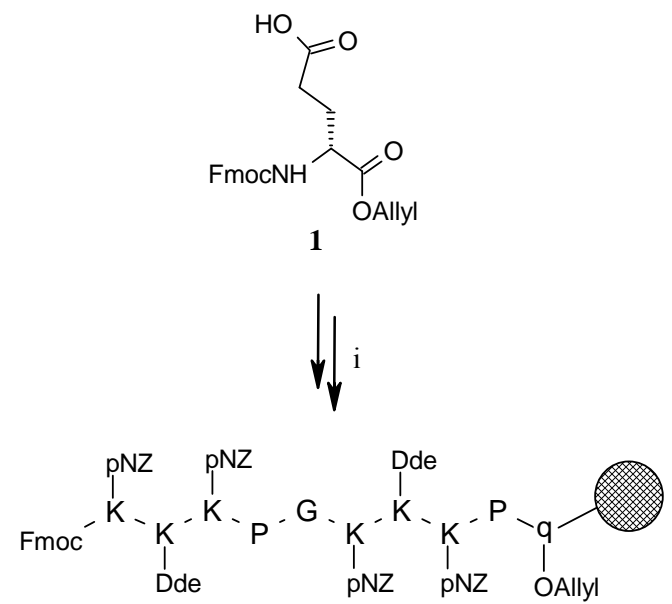

2
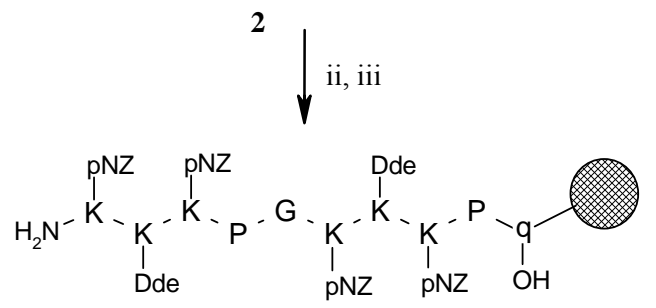

3
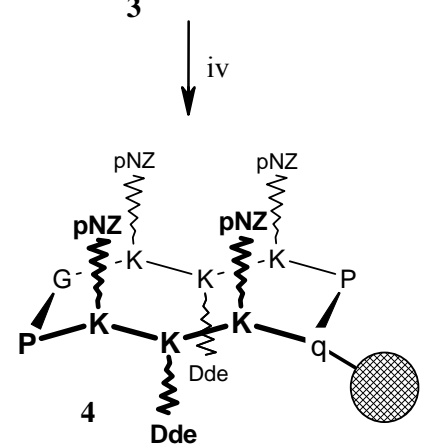

Fig. (2). Synthesis of orthogonally protected cyclodecapeptide 4.

Reagents and conditions: i: Standard Fmoc strategy; ii: $\mathrm{Pd}_{(}\left(\mathrm{Ph}_{3} \mathrm{P}_{4}\right.$, $\mathrm{PhSiH}_{3}, \mathrm{CH}_{2} \mathrm{Cl}_{2}$, r.t., $2 \times 20 \mathrm{~min}$; iii: $20 \%$ piperidine, DMF, r.t., $3 \times 10$ min; iv: PyAOP, DIPEA, DMF, r.t., $2 \times 1$ h.

Further functionalization of the cyclic template was realized sequentially, after regioselective removal of orthogonal protecting groups (Fig. 3). As for cyclization step, the completeness of each reaction was controlled by TNBS and Kaiser tests. Dde was first deprotected by treatment of cylodecapaptide 4 with hydrazine (2\%) in DMF [22]. This provides two free lysine side chains pointing on the lower addressable domain of the template. To ensure the immobilization or the detection of these molecules for biological studies, we coupled biotins to the latter lysine side chains by using the standard coupling conditions to obtain biotinylated peptide 5 .

As mentioned above, our procedure to graft carbohydrates to the template follows an oxime-based strategy. This requires the incorporation of an aminooxy function into sugar derivatives as well as complementary electrophilic function on the template $[18,30]$. In order to prevent oxida- 
tion of biotin during the formation of aldehydes from serine residues [38], we introduced levulinic acid to provide a methyl ketone, despite the moderate coupling yields that we observed previously with oxyamines [18]. The removal of pNZ group from $\mathbf{5}$ was realized by treatment of the resin with a solution of $\mathrm{SnCl}_{2} 2 \mathrm{M}$, Phenol $0.01 \mathrm{M}$, AcOH $1.6 \mathrm{mM}$ in DMF to release four lysine side chains [21]. The subsequent reaction of this compound with pentafluorophenol levulinic ester provided the biotinylated RAFT molecule presenting clustered ketones 6 . Surprisingly, the analysis of an aliquot of the crude product $\mathbf{6}$ has shown a broad peak by HPLC, whereas the expected signal was found by mass spectrometry. By contrast, a clean reaction mixture was obtained after pNZ deprotection from the linear peptide $\mathbf{2}$, suggesting that impurities resulting from this reaction could be trapped by the cyclic core of the template. Similar broad signals were observed in each following HPLC analysis.

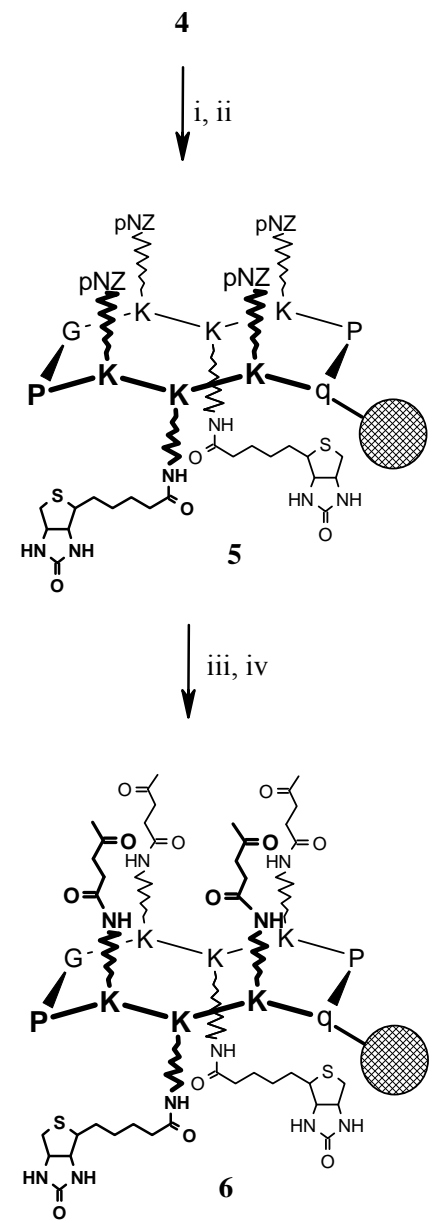

Fig. (3). Synthesis of cyclodecapeptide displaying ketones and biotins 6.

Reagents and conditions: i: 2\% Hydrazine, DMF, r.t., 4x10 min; ii: Biotin, PyBOP, DIPEA, DMF, r.t., 1 h; iii: $\mathrm{SnCl}_{2} 2 \mathrm{M}$, Phenol $0.01 \mathrm{M}, \mathrm{AcOH}$ 1.6mM, DMF, r.t., $3 \times 2$ h; iv: Pfp-levulinic ester [18], DIPEA, DMF, r.t., $2 \times 1$ h.

Aminooxy carbohydrates 9-13 have been synthesized following the previously reported procedure (Fig. 4) [29, 33]. As illustrated for the $\alpha \mathrm{Gal}$ series, the galactosyl fluoride 7 protected with benzyl groups was treated with boron trifluoride-diethyl etherate and triethylamine in dichlor- methane in the presence of $N$-hydroxyphthalimide as precursor of aminooxy function. Due to the non-participation of benzyl group at carbon 2, this glycosylation afforded the expected alpha anomer $\mathbf{8}$ as major product and the corresponding beta diastereoisomer which were both isolated by silica gel chromatography in $62 \%$ and $25 \%$ yield respectively. After deprotection of benzyl by hydrogenolysis, removal of phthalimide group with methylhydrazine then purification by precipitation, the pure aminooxy $\alpha$-Gal derivative 9 was obtained with $55 \%$ yield. A similar strategy was followed to prepare $\beta$-Gal 10, $\alpha$-Man 11, $\alpha$-GalNAc 12 and $\beta$ Lac moieties 13. Each of the above carbohydrates has been unambiguously analyzed by NMR spectroscopy and mass spectrometry.

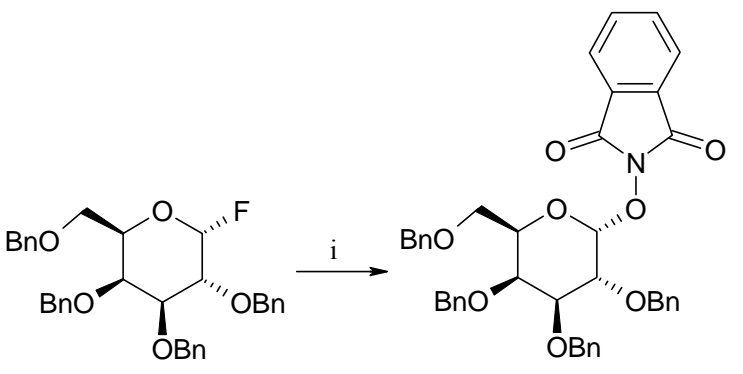

7

$8(62 \%+25 \%$ of $\beta$ anomer $)$<smiles>CC#CC</smiles>

$9\left(55 \% ; \alpha-\mathrm{D}-\mathrm{Gal}-\mathrm{ONH}_{2}\right)$<smiles>NOC1O[C@H](CO)[C@@H](O)[C@H](O)[C@H]1O</smiles><smiles>NO[C@H]1O[C@H](CO)[C@@H](O)[C@H](O)[C@H]1O</smiles>

$10\left(\beta-\mathrm{D}-\mathrm{Gal}-\mathrm{ONH}_{2}\right)$

$11\left(\alpha-\mathrm{D}-\mathrm{Man}-\mathrm{ONH}_{2}\right)$<smiles>CN[C@H]1[C@@H](ON)O[C@H](CO)[C@@H](O)[C@@H]1O</smiles>

$\left.12(\alpha-\mathrm{D}-\text { GalNAc-ONH})_{2}\right)$<smiles>NO[C@H]1O[C@H](CO)[C@@H](O[C@@H]2O[C@H](CO)[C@@H](O)[C@H](O)[C@H]2O)[C@H](O)[C@H]1O</smiles>

$13\left(\beta-\mathrm{D}-\mathrm{Lac}-\mathrm{ONH}_{2}\right)$

Fig. (4). General strategy for the synthesis of aminooxy carbohydrates 9-13.

Reagents and conditions: $\mathrm{i}: \mathrm{BF}_{3} \cdot \mathrm{Et}_{2} \mathrm{O}$, triethylamine, $\mathrm{CH}_{2} \mathrm{Cl}_{2}, \mathrm{r}$. t., $1 \mathrm{~h}$; ii: $\mathrm{H}_{2}, \mathrm{Pd} / \mathrm{C}, \mathrm{MeOH}, 1$ atm, r. t. 4 h; iii: methylhydrazine, EtOH, r. t., 12 h.

The chemoselective conjugation of either alpha or beta aminooxy sugars was finally performed on the solid support using a 4-fold excess of sugar per anchoring site in a mixture of DMF/AcOH/H2O (1/1/0.1). The resin was stirred overnight at room temperature then the excess of unreacted sugars was recovered by filtration. After released from the support by treatment with TFA, precipitation in diethyl ether 
and semi-preparative purification, pure biotinylated glycoclusters 14-18 were obtained, as determined with the initial resin loading, with a $10 \%$ estimated overall yield. No significant difference of reactivity depending on the nature of aminooxy carbohydrate was observed. Moreover, the final acidic treatment had no incidence on oxime bond stability as expected and confirmed by mass spectroscopy analysis (Table 1).

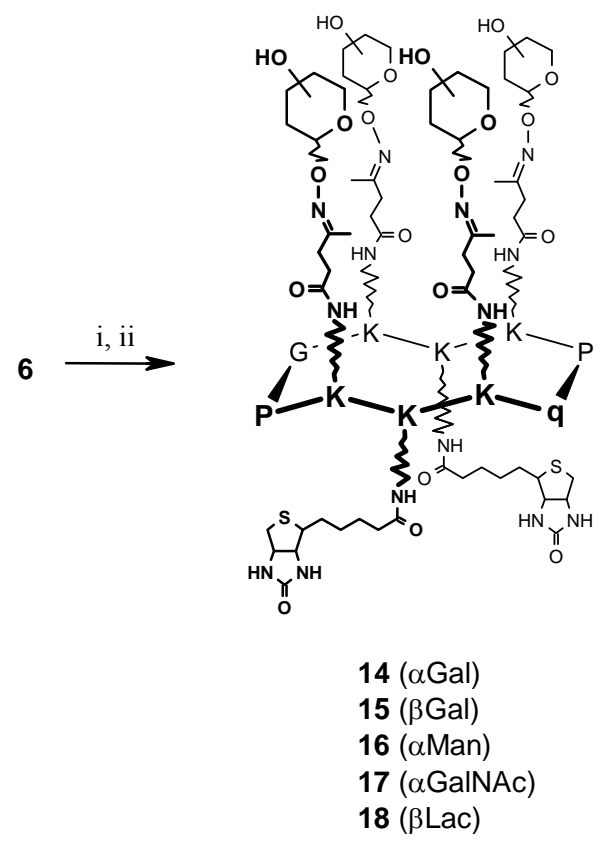

Fig. (5). Chemoselective coupling of sugars and release of glycoclusters 14-18 from the support.

Reagents and conditions: i: 9-13, DMF/AcOH/ $\mathrm{H}_{2} \mathrm{O}(1 / 1 / 0.1)$, r.t., $12 \mathrm{~h}$; ii: TFA, r. t., $1 \mathrm{~h}$.

Table 1. Analytical Data of Glycoclusters 13-16

\begin{tabular}{|c|c|c|c|}
\hline Glycocluster & $\mathbf{R}_{\mathbf{t}}{ }^{a}$ & $\mathbf{M}_{\text {calcd. }}$ & $\mathbf{~ m / \mathbf { z } ^ { b }}$ \\
\hline \hline $\mathbf{1 4}$ & 18.3 & 2700.3 & {$[\mathrm{M}+3 \mathrm{H}]^{3+}=901.4[\mathrm{M}+2 \mathrm{H}]^{2+}=1352.0$} \\
$\mathbf{1 5}$ & 18.7 & 2700.3 & {$[\mathrm{M}+3 \mathrm{H}]^{3+}=901.2[\mathrm{M}+2 \mathrm{H}]^{2+}=1352.4$} \\
$\mathbf{1 6}$ & 18.5 & 2700.3 & {$[\mathrm{M}+3 \mathrm{H}]^{3+}=901.4[\mathrm{M}+2 \mathrm{H}]^{2+}=1352.9$} \\
$\mathbf{1 7}$ & 18.9 & 2864.4 & {$[\mathrm{M}+3 \mathrm{H}]^{3+}=956.2[\mathrm{M}+2 \mathrm{H}]^{2+}=1434.3$} \\
$\mathbf{1 8}$ & 18.9 & 2248.5 & {$[\mathrm{M}+3 \mathrm{H}]^{3+}=1117.8[\mathrm{M}+2 \mathrm{H}]^{2+}=1679.2$} \\
\hline
\end{tabular}

${ }^{a}$ Reverse-Phase HPLC retention time (Rt) are given in minutes: linear gradient 95:5 A:B to 40:60 A:B in $30 \mathrm{~min}$ (Column: nucleosil $100 \AA 5 \mu \mathrm{m} \mathrm{C} 18$ particles, 250x4.6 $\mathrm{mm}$; solvent B: $0.09 \%$ TFA in $90 \%$ acetonitrile and solvent A: $0.09 \%$ TFA; flow: 1 $\mathrm{mL} / \mathrm{min}$; detection: $\lambda=214 \mathrm{~nm}$ and $250 \mathrm{~nm}$ ).

${ }^{b}$ Mass spectrometry analysis was performed by electrospray ionisation method in positive mode.

\section{CONCLUSION}

In this manuscript, we report the fully solid-phase synthesis of various well-defined, biotinylated glycoclusters 1418 which can be readily immobilized on streptavidin-coated surfaces for studying protein-carbohydrate interactions. In comparison with our previous protocol which requires several time-consuming HPLC purifications, this polymersupported methodology, including both cyclization and topo- logical functionalization of the template, represents a significant improvement. Having in hand each carbohydrate and amino acid building blocks, this synthesis occurs indeed within a few days, besides suggesting that this protocol could be extended by using an automatic synthesizer. Consequently, we assume that this approach represents a prerequisite for a rapid optimization of glycocluster structures through combinatorial techniques. This latter point is currently under investigation in the laboratory and will be reported in due course.

\section{ACKNOWLEDGMENTS}

This work was supported by the Association pour la Recherche contre le Cancer (ARC), the Centre National pour la Recherche Scientifique (CNRS), the Université Joseph Fourier (UJF) and COST D34.

\section{REFERENCES}

[1] Varki A. Biological roles of oligosaccharides: all of the theories are correct. Glycobiology 1993; 3(2): 97-130.

[2] Dwek RA. Glycobiology: toward understanding the function of sugars. Chem Rev 1996; 96(2): 683-720.

[3] Bertozzi CR, Kiessling L. Chemical Glycobiology. Science 2001; 291(5512): 2357-64

[4] Lee YC, Lee RT. Carbohydrate-protein interactions: basis of glycobiology. Acc Chem Res 1995; 28(8): 321-7.

[5] Mammen M, Choi SK, Whitesides GM. Polyvalent interactions in biological systems: implications for design and use of multivalent ligands and inhibitors. Angew Chem Int Ed 1998; 37(20): 2754-94.

[6] Lundquist JJ, Toone EJ. The cluster glycoside effect. Chem Rev 2002; 102(2): 555-78.

[7] Raman R, Raguram S, Venkataraman G, Paulson JC, Sasisekharan R. Glycomics : an integrated systems approach to structurefunction relationships of glycans. Nat Methods 2005; 2(11); $817-$ 24

[8] Pilobellol KT, Mahal LK. Deciphering the glycocode: the complexity and analytical challenge of glycomics. Curr Opin Chem Biol 2007; 11(3): 1-6.

[9] Ratner DM, Adams EW, Disney MD, Seeberger PH. Tools for glycomics: mapping interactions of carbohydrates in biological systems. ChemBioChem 2004; 5(10): 1375-83

[10] Baldini L, Casnati A, Sansone F, Ungaro R. Calixarene-based multivalent ligands. Chem Soc Rev 2007; 36(2): 254-66.

[11] Ortiz Mellet C, Defaye J, Garcia Fernandez JM. Multivalent cyclooligosaccharides: versatile carbohydrate clusters with dual role as molecular receptors and lectin ligands. Chem Eur J 2002; 8(9): 1982-90.

[12] Niederhafner P, Sebestik J, Jezek J. Glycopeptide dendrimers. J Pept Sci 2008; 14(1): 2-43.

[13] Boturyn D, Defrancq E, Dolphin GT, Garcia J, Labbé P, Renaudet O, Dumy P. RAFT nano-constructs: surfing to biological applications. J Pept Sci 2008; 14(2): 224-40.

[14] Singh Y, Renaudet O, Defrancq E, Dumy P. Preparation of a multitopic glycopeptide-oligonucleotide conjugate. Org Lett 2005; 7(7): 1359-62.

[15] Renaudet O, BenMohamed L, Dasgupta G, Bettahi I, Dumy P. Towards self-adjuvanting multivalent $\mathrm{B}$ and $\mathrm{T}$ cell epitopes synthetic glyco-lipopeptide cancer vaccine. ChemMedChem 2008; Published online Jan 182008.

[16] Boturyn D, Coll JL, Garanger E, Favrot MC, Dumy P. Template assembled cyclopeptides as multimeric system for integrin targeting and endocytosis. J Am Chem Soc 2004; 126(18): 5730-9.

[17] Razkin J, Josserand V, Boturyn D, et al. Activatable fluorescent probes for tumour-targeting imaging in live mice. ChemMedChem 2006; 1(10): 1069-72.

[18] Renaudet O, Dumy P. Synthesis of multitopic neo-glycopeptides displaying recognition and detection motifs. Bioorg Med Chem Lett 2005; 15(15): 3619-22.

[19] Wilczewski M, Van der Heyden A, Renaudet O, Dumy P, CocheGuérente L, Labbé P. Promotion of sugar-lectin recognition through multiple sugar presentation offered by Regioselectively 
Addressable Functionalized Templates (RAFT). Org Bioorg Chem 2008; 6(6): 1114-22.

[20] Mutter M, Dumy P, Garrouste P, et al. Template assembled synthetic proteins (TASP) as functional mimetics of proteins. Angew Chem Int Ed 1996; 35(13/14): 1482-85.

[21] Hocker MD, Caldwell CG, Macsata RW, Lyttle MH. p-nitrobenzyl side-chain protection for solid-phase synthesis. Peptide Res 1995; 8(10): 310-15.

[22] Bycroft BW, Chan WC, Chabra SR, Hone ND. A novel lysineprotecting procedure for continuous flow solid phase synthesis of branched peptides. J Chem Soc Chem Commun 1993; 9: 778-9.

[23] Hang HC, Bertozzi CR. Chemoselective approaches to glycoprotein assembly. Acc. Chem Res 2001; 34(9): 727-36.

[24] Langenhan JM, Thorson JS. Recent carbohydrate-based chemoselective ligation applications. Curr Org Synth 2005; 2(1): 59-81.

[25] Kimmerlin T, Seebach D. 100 years of peptide synthesis: ligation methods for peptide and protein synthesis with applications to $\beta$ peptide assemblies. J Pept Res 2005; 65(2): 229-60.

[26] Peri F, Nicotra F. Chemoselective ligation in glycochemistry. Chem Comm 2004; 6: 623-27.

[27] Grochowski E, Jurczak J. A new class of monosaccharide derivatives: O-phthalimidohexoses. Carbohydrate Res 1976; 50(2): C15C16.

[28] Cao S, Tropper FD, Roy R. Stereoselective phase transfer catalyzed syntheses of glycosyloxysuccinimides and their transformations into glycoprobes. Tetrahedron 1995; 51(24): 6679-86.

[29] Renaudet O, Dumy P. Expedient synthesis of amino-oxylated carbohydrates for chemo-selective access of glycoconjugates. Tetrahedron Lett 2001; 42(43): 7575-78.

[30] Renaudet O, Dumy P. Chemoselectively template-assembled glycoconjugates as mimics for multivalent presentation of carbohydrates. Org Lett 2003; 5(3): 243-6.
[31] Rodriguez EC, Marcaurelle LA, Bertozzi CR. Aminooxy-, hydrazide-, and thiosemicarbazide-functionalized saccharides: versatile reagents for glycoconjugate synthesis J Org Chem 1998; 63(21): 7134-5.

[32] Coste J, Le-Nguyen, D, Castro B. PyBOP: a new peptide coupling reagent devoid of toxic byproduct. Tetrahedron Lett 1990; 31(2): 205-8.

[33] Renaudet O, Dumy P. On-bead synthesis and binding assay of chemoselectively template-assembled multivalent neoglycopeptides. Org Biomol Chem 2006; 4(13): 2628-36.

[34] Thieriet N, Alsina J, Girald E, Guibé F, Albericio F. Use of Allocamino acids in solid-phase peptide synthesis. Tandem deprotectioncoupling reactions using neutral conditions. Tetrahedron Lett 1997; 38(41): 7275-78.

[35] Kaiser E, Colescott RL, Bossinger CD, Cook PI. Color test for detection of free terminal amino groups in the solid-phase synthesis of peptides. Anal Biochem 1970; 34(2): 595-8.

[36] Hancock WS, Battersby JE. A new micro-test for the detection of incomplete coupling reactions in solid-phase peptide synthesis using 2,4,6-trinitrobenzenesulfonic acid. Anal Biochem 1976; 71(1): 260-4.

[37] Albericio F, Cases M, Alsina J, Triolo SA, Carpino LA, Kates SA. On the use of PyAOP, a phosphonium salt derived from HOAt, in solid-phase peptide synthesis. Tetrahedron Lett 1997; 38(27): 4853-56.

[38] Geoghegan KF, Stroh JG. Site-directed conjugation of nonpeptide groups to peptides and proteins via periodate oxidation of a 2 amino alcohol. Application to modification at N-terminal serine. Bioconjugate Chem 1992; 3(2): 138-46. 